\title{
Nível de atividade física em pessoas com diabetes mellitus tipo 2
}

\author{
Level of physical activity in people with type-2 diabetes mellitus
}

\section{Nivel de actividad física en personas con diabetes mellitus tipo 2}

\author{
Flávia Cristiane Kolchraiber ${ }^{1}{ }^{(}$, Jusceline de Souza Rocha ${ }^{2} \mathbb{D}^{-}$, Denise Jovê César ${ }^{3} \mathbb{D}^{\text {, }}$ \\ Odete de Oliveira Monteiro ${ }^{4}{ }^{\circledR}$, Giovana Andrade Frederico ${ }^{5}{ }^{\circledR}$, Mônica Antar Gamba ${ }^{6}{ }^{\mathbb{D}}$
}

\section{Histórico}

Recibido:

15 de febrero de 2018

Aceptado:

05 de abril de 2018

1 Mestre em Ciências da Saúde pela Universidade Federal de São Paulo, São Paulo, Brasil.

2 Enfermeira Pela Escola Paulista de Enfermagem, Universidade Federal de São Paulo, São Paulo, Brasil.

3 Mestre em Educação Universidade Federal de Mato Grosso do Sul, Mato Grosso do Sul, Brasil.

4 Doutora em Enfermagem, Universidade Federal de São Paulo (UNIFESP), São Paulo, Brasil.

5 Enfermeira. Escola Paulista de Enfermagem, Universidade Federal de São Paulo, São Paulo, Brasil. Autor de Correspondência: $\quad$ E-mail: giovanaafrederico@gmail.com 6 Doutora em Saúde Pública, Universidade de São Paulo (USP) Brasil Professora Associada do Departamento de Enfermagem em Saúde Coletiva da Escola Paulista de Enfermagem da Universidade Federal de São Paulo, São Paulo - SP, Brasil.
Introdução: $\mathrm{O}$ estilo de vida tem se destacado como importante causa para as principais doenças crônicas, como o diabetes mellitus tipo 2, por causar alterações fisiológicas crônicas que tendem a se agravar à medida que o indivíduo envelhece. Comparou-se o nível de atividade física realizada por pessoas com diabetes mellitus tipo 2 na atenção básica e unidade especializada. Materiais e Métodos: Estudo descritivo, de corte transversal, com análise comparativa. Aplicaram-se questionários semiestruturado e o validado Internacional de Atividade Física, submetidos e comparados por análise estatística com os testes do Qui-quadrado de Pearson e $t$ student. Resultados: A idade média dos usuários foi 59 anos, predominância sexo feminino e não praticantes de atividade física. Valores glicêmicos obtidos pelo exame da hemoglobina glicada foram, $8,1 \%$ em centro de referência e $9,6 \%$ em unidade básica de saúde $(p=0,017)$, diferenciais de controle metabólico e distintas realidades de atendimento. Discussão: O nível de atividade física aliado a mudanças no estilo de vida e adesão terapêutica, é parte fundamental para o controle do diabetes e prevenção de complicações, devendo ser encorajadas pelos profissionais da saúde. Conclusoes: Melhor desempenho no nível de atividade física e melhor controle glicêmico de pessoas acompanhadas em centro especializado. Deve-se considerar ações de educação à saúde na perspectiva do cuidado integral na Rede de Atenção à Saúde, independente do tipo de serviço, como potencializadoras para o automonitoramento e controle do diabetes mellitus.

Palavras chave: Diabetes Mellitus; Atividade Motora; Enfermagem em Saúde Comunitária.

Introduction: Lifestyle has emerged as an important cause of major chronic diseases, such as type- 2 diabetes mellitus, because it causes chronic physiological changes that tend to worsen as individual age. The study compared the level of physical activity performed by people with type- 2 diabetes mellitus in basic care and specialized unit. Materials and Methods: Descriptive, cross-sectional study with comparative analysis. Semistructured questionnaires and the internationally validated Physical Activity questionnaire were applied, submitted and compared through statistical analysis with Pearson's chi-square and Student's t tests. Results: The average age of the users was 59 years, with predominance of females and those not engaging in physical activity. Glycemic values obtained by examining glycated hemoglobin were: $8.1 \%$ in a reference center and $9.6 \%$ in a basic health unit $(p=0.017)$, differentials of metabolic control and different care realities. Discussion: The level of physical activity, combined with changes in lifestyle and therapeutic adherence, is a fundamental part of diabetes control and prevention of complications and should be encouraged by health professionals. Conclusions: Better performance was noted in the level of physical activity and better glycemic control of individuals monitored at a specialized center. Health education actions should be considered within the perspective of comprehensive care in the Health Care Network, regardless of the type of service, as potential for self-monitoring and control of diabetes mellitus. Key words: Diabetes Mellitus; Motor Activity; Community Health Nursing.

Resumen

Introducción: El estilo de vida se ha destacado como una importante causa para las principales enfermedades crónicas, como la diabetes mellitus tipo 2, por causar alteraciones fisiológicas crónicas que tienden a agravarse a medida que el individuo envejece. Se comparó el nivel de actividad física realizada por personas con diabetes mellitus tipo 2 en la atención básica y en unidad especializada. Materiales y Métodos: Estudio descriptivo de corte transversal con análisis comparativo. Se aplicaron cuestionarios semiestructurados y el validado Internacional de Actividad Física, sometidos y comparados por análisis estadístico con las pruebas del Chi-cuadrado de Pearson y $t$ student. Resultados: La edad media de los usuarios fue de 59 años, predominancia del sexo femenino y no practicantes de actividad física. Los valores glucémicos obtenidos por el examen de la hemoglobina glicosilada fue de $8.1 \%$ en centro de referencia y $9.6 \%$ en unidad básica de salud $(p=0,017)$, diferenciales de control metabólico y distintas realidades de atención. Discusión: El nivel de actividad física aliado a cambios en el estilo de vida y adhesión terapéutica es parte fundamental para el control de la diabetes y prevención de complicaciones, debiendo ser estimuladas por los profesionales de la salud. Conclusiones: Mejor desempeño en el nivel de actividad física y mejor control glucémico de personas acompañadas en centro especializado. Se debe considerar acciones de educación a la salud en la perspectiva del cuidado integral en la Red de Atención a la Salud, independiente del tipo de servicio, como potencializadoras para el automonitoramiento y control de la diabetes mellitus.

Palabras clave: Diabetes Mellitus; Actividad Motora; Enfermería en Salud Comunitaria.

Como citar este artigo: Kolchraiber FC, Rocha JS, César DJ, Monteiro OO, Frederico GA, Gamba MA. Nivel de atividade fisica em pessoas com diabetes mellitus tipo 2. Rev Cuid. 2018; 9(2):2105-16. http://dx.doi.org/10.15649/cuidarte.v9i2.512

(c) (i) () 2018 Universidad de Santander. Este es un artículo de acceso abierto, distribuido bajo los términos de la licencia Creative Commons Attribution (CC BY-NC 4.0), que permite el uso ilimitado, distribución y reproducción en cualquier medio, siempre que el autor original y la fuente sean debidamente citados. 


\section{INTRODUÇÃO}

As mudanças trazidas pela Era Industrial e atualmente Era digital, as pessoas passaram a ter acesso a bens materiais e máquinas que lhes proporcionaram mais facilidade e rapidez para a realização das atividades diárias, resultando, com isso, em uma redução na prática de atividade física da população em geral. O sedentarismo e a má alimentação têm se destacado como importante causa para o surgimento das principais doenças crônicas, em especial, o diabetes mellitus tipo 2 $(\mathrm{DM} 2)^{1}$.

A prevalência de pessoas com diabetes em todo o mundo mais do que duplicou nos últimos 20 anos, mantendo níveis epidêmicos diferenciados. Uma das características mais preocupantes deste rápido aumento é o aparecimento de DM2 em crianças, adolescentes e adultos jovens ${ }^{2}$. No Brasil, as regiões mais desenvolvidas economicamente apresentam maior prevalência de diabetes mellitus e são vários os fatores ambientais relacionados a esse aumento, dentre eles o sedentarismo e a obesidade ${ }^{3}$.

O DM2 promove alterações fisiológicas irreversíveis, que acompanham o processo de envelhecimento do indivíduo, e somado ao seu estilo de vida, torna esta grave situação um desafio a ser vencido pelos usuários e profissionais de saúde.

Estudos epidemiológicos demonstram a relação inversamente profissional entre o nível de atividade física e o surgimento de complicações crônicas, bem como diversos fatores de risco cardiovascular em indivíduos com DM2.
Neste contexto, alterações fisiológicas não podem ser associadas somente ao processo de envelhecimento per si, mas sim, aos padrões de estilo de vida ${ }^{4}$. Destacam-se dentre eles a redução da atividade física, distúrbios do sono, má alimentação e o aumento da obesidade central. Estes são fatores de risco independentes para o desenvolvimento de complicações crônicas em indivíduos com DM2 e, quando associados, potencializam sua progressão das mesmas ${ }^{5,6}$.

O DM2 é uma síndrome metabólica de origem multifatorial. Se não tratado e bem controlado, produz, no transcorrer do tempo, danos severos e potencialmente fatais, como o infarto agudo do miocárdio, acidente vascular cerebral, cegueira, impotência, acometimento renal, úlceras e amputações de membros inferiores. No caminho reverso, as complicações decorrentes da cronicidade podem ser evitadas e é possível que o paciente com diabetes tenha qualidade de vida satisfatória, a partir de uma vida saudável e com suporte social $^{7-9}$. Estudos mostram que o risco de desenvolvimento do DM2 aumenta proporcionalmente ao índice de massa corporal (IMC), esse número é contrário quando há aumento da intensidade e o período da atividade física, considerando o consumo calórico semanal ${ }^{10,11}$.

O tratamento do diabetes mellitus é associado à disciplina e ao comprometimento do paciente implicando na mudança de comportamentos. A atividade física exerce um papel de fundamental importância na qualidade de vida da pessoa com DM2 e deve ser realizada de forma regular, sistemática e orientada por um profissional de educação física, abordando as reais necessidades 
do indivíduo, avaliando e prescrevendo os exercícios físicos ideais para cada situação.

A relação entre o aumento dos níveis de atividade física e a menor frequência de complicações crônicas sugere que a atividade física exerce um papel protetor para indivíduos com DM2 $2^{12-13}$.

Os benefícios e efeitos da atividade física para o indivíduo com DM2 são observados logo no inicio da prática e horas após o seu término. Com a prática do exercício físico é possível aumentar cerca de 20 vezes a utilização de glicose pelo músculo, aumentando a sensibilidade à insulina e auxiliando na redução dos níveis de glicemia para a faixa normal. Remetendo à recomendação da prática da atividade física conforme preconiza a Organização Mundial de Saúde (OMS) de 150 minutos de atividades semanais para adultos ${ }^{2,12}$.

No Brasil, utiliza-se o Questionário Internacional de Atividade Física (IPAQ), validado e adotado para a classificação do nível de atividade física realizada, ordenado em cinco tipos: muito ativo, ativo, irregularmente ativo tipo $\mathrm{A}$, irregularmente ativo tipo B e sedentário ${ }^{13,14}$. A justificativa desta investigação se dá pela escassez de dados epidemiológicos destinados a quantificar o nível de atividade física em indivíduos com DM2 atendidos nas instituições públicas de saúde, refletindo a importância de mensurar o nível de atividade física praticado pelo paciente durante as avaliações realizadas pelas equipes de enfermagem na atenção à saúde.

Assim, este estudo teve por objetivo comparar os níveis de atividade física realizada por pessoas com diabetes mellitus tipo 2 (DM2) relacionado ao modelo de atenção à saúde oferecido por tipo de serviço, em atenção básica e unidade especializada.

\section{MATERIAIS E MÉTODOS}

Trata-se de um estudo descritivo, de coorte transversal de análise comparativa respeitando os critérios estabelecidos pelo Strengthening the Reporting of Observational Studies in Epidemiology-STROBE ${ }^{15}$, baseando-se em dois serviços com modelos distintos de atenção à saúde, no período de maio a julho de 2014. A amostra populacional estimada foi de 115 usuários, porém após aplicação de critério de inclusão e exclusão, obteve-se o total de 62 participantes.

Os dados foram coletados em duas instituições de saúde dos municípios de São Paulo e Embu das Artes. Sendo uma localizada em região central e outra periférica. A primeira com atendimento secundário, Centro de Especialidades em Diabetes Mellitus (CEDM). A segunda com atendimento primário, Unidade Básica de Saúde (UBS) com modelo de Estratégia de Saúde da Família (ESF).

Foram estipulados como critérios de inclusão: diagnóstico por DM2 (CID E11) há mais de 5 anos, idade superior a 45 anos, IMC superior a $25 \mathrm{Kg} / \mathrm{m}^{2}$, exame laboratorial de hemoglobina glicada (HbA1c) superior a 7\%. A coleta de dados foi realizada por meio de dois instrumentos: questionário semi estruturado contendo dados de identificação e variáveis clínicas. E o IPAQ, questionário proposto pela Organização Mundial da Saúde, para ser utilizado como instrumento 
mundial para classificação do nível de atividade física populacional, neste estudo utilizado na versão curta ${ }^{16}$. A classificação é obtida pela somatória da frequência e da duração das atividades físicas realizadas por pelo menos 10 minutos contínuos durante a última semana seguindo o seguinte padrão:

Sedentário - Não realiza nenhuma atividade física por pelo menos 10 minutos contínuos durante a semana;

Insuficientemente Ativo - Consiste em classificar os indivíduos que praticam atividades físicas por pelo menos 10 minutos contínuos por semana, porém de maneira insuficiente para ser classificado como ativos. Para classificar os indivíduos nesse critério, são somadas a duração e a freqüência dos diferentes tipos de atividades (caminhadas + moderada + vigorosa). Essa categoria dividese em dois grupos: Insuficientemente Ativo A Realiza 10 minutos contínuos de atividade física, seguindo pelo menos um dos critérios citados: freqüência - 5 dias/semana ou duração - 150 minutos/semana; Insuficientemente Ativo B Não atinge nenhum dos critérios da recomendação citada nos indivíduos insuficientemente ativos A; Ativo - Cumpre as seguintes recomendações: a) atividade física vigorosa $-\geq 3$ dias/semana e $\geq 20$ minutos/sessão; b) moderada ou caminhada $-\geq 5$ dias/semana e $\geq 30$ minutos/sessão; c) qualquer atividade somada: $\geq 5$ dias/semana e $\geq 150 \mathrm{~min} /$ semana;

Muito Ativo - Cumpre as seguintes recomendações: a) vigorosa $-\geq 5$ dias/semana e $\geq 30 \mathrm{~min} /$ sessão; b) vigorosa $-\geq 3 \mathrm{dias} /$ semana e $\geq 20 \mathrm{~min} / \mathrm{sessão}+$ moderada e ou caminhada $\geq$ 5 dias/ semana e $\geq 30 \mathrm{~min} /$ sessão $^{13,14}$.
O projeto de pesquisa foi submetido ao Comitê de Ética e Pesquisa, para apreciação sendo o mesmo aprovado pelo parecer consubstanciado $n^{\circ} 19668 / 12$. Às coordenadoras dos serviços de saúde foi solicitado parecer do projeto e os participantes aderiram ao mesmo, assinando o termo de consentimento livre e esclarecido, cumprindo todos os requisitos éticos exigidos pela Portaria 510/2016 do Conselho Nacional de Saúde que dispõe sobre pesquisa envolvendo seres humanos.

A análise e apresentação descritiva dos dados foram realizadas por meio de gráficos e tabelas expondo as frequências simples, percentagens, médias, desvios-padrão entre outras análises descritivas.

Os dados foram compostos por variáveis do tipo qualitativa (local, sexo, atividade física) e quantitativa (idade, peso, altura, IMC, HbA1c). Para interpretação de comparações e inferência foram usados os seguintes testes estatísticos: Teste Qui-quadrado de Pearson para análise de associação,com significância adotada 5\%. Teste $t$ de student para comparação das medias entre os dois grupos,valor de $\mathrm{p}$ for menor que a significância adotada ${ }^{17}$.

\section{RESULTADOS}

Dos 62 participantes a predominância de 63,2\% do sexo feminino em ambos serviços de saúde, havendo uma maior evidencia no CEDM com $67,5 \%$. A média de idade das pessoas atendidas foi maior no CEDM, de 60 anos, já na UBS, foi de 58 anos conforme (Figura 1). 


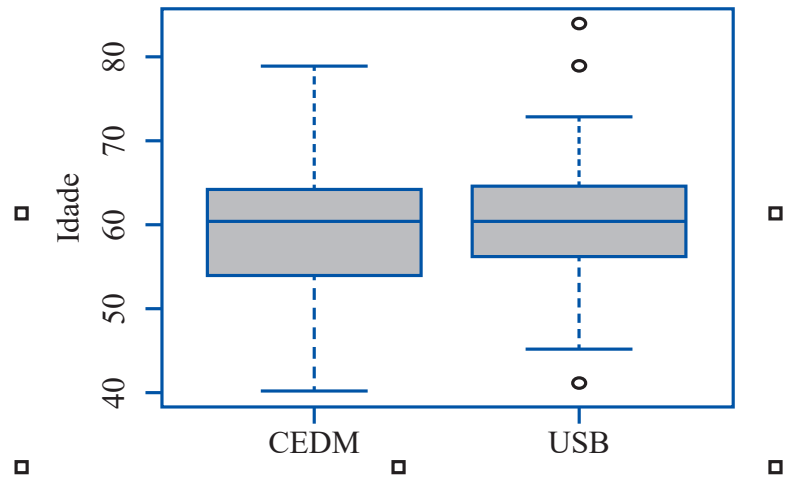

Figura 1. Distribuição das pessoas com DM

CEDM - Centro de Especialidades em Diabetes Mellitus; UBS-Unidade Básica de Saúde.

A partir da apresentação dos dados coletados, é possível observar que o número de pessoas com IMC dentro dos parâmetros normais é superior no CEDM, quando comparados com a UBS. A análise dos dados mostrou também que as pessoas atendidas no CEDM são diagnosticadas precocemente em relação à faixa etária, quando comparadas com a UBS. Em média as pessoas são diagnosticadas no CEDM com 45 anos, já na
UBS a média foi de 49 anos.

Quanto aos níveis de HbA1c avaliados nas pessoas atendidas, apresentaram uma média de HbA1c de $8,1 \%$ identificados no CEDM, enquanto os participantes na UBS apresentaram uma média de $9.6 \%$ e observou se diferença estatisticamente significativa conforme (Tabela 1).

Tabela 1. Distribuição das pessoas com Diabetes Mellitus tipo 2 por local de atendimento, segundo dosagem de HbA1c. São Paulo, 2014

\begin{tabular}{|c|c|c|c|c|}
\hline \multirow{2}{*}{ Localidade } & \multicolumn{5}{c}{ HbA1c } \\
\cline { 2 - 5 } & Média & Desvio Padrão & CV(\%) & N \\
\hline UBS & 9,64 & 2,90 & $30,1 \%$ & 14 \\
\hline CEDM & 8,08 & 1,92 & $23,8 \%$ & 33 \\
\hline
\end{tabular}

$p=0,017$

HbAlc-hemoglobina glicada; CV - Coeficiente de variação;UBS - Unidade Básica de Saúde; CEDM - Centro de Especialidades em Diabetes Mellitus.

Fonte: dados primários de pacientes com diabetes mellitus de unidades de saúde

Analisando as variáveis categóricas de atividades físicas e IMC observa-se que quanto mais acima do peso o paciente se encontra, menor o nível de atividade física, 53,3\% da população estudada foi classificada como sedentária, destes $46 \%$ com obesidade moderada. 
$\mathrm{O}$ cruzamento das categorias atividade física x IMC, mostrou que pessoas que se encontram com o peso acima dos parâmetros normais, são em sua maioria sedentários, mas também são praticantes de atividades físicas conforme (Tabela 2).

Tabela 2. Distribuição de pessoas com Diabetes Mellitus tipo 2 por IMC, segundo nível de atividade física. São Paulo, 2014

\begin{tabular}{|c|c|c|c|c|c|}
\hline \multirow{2}{*}{\multicolumn{2}{|c|}{ Faixa do IMC }} & \multicolumn{3}{|c|}{ Atividade } & \multirow{2}{*}{ Total } \\
\hline & & Sedentário & Ativo & Muito Ativo & \\
\hline \multirow{2}{*}{ Normalidade } & $\mathrm{N}$ & 10 & 6 & 2 & 18 \\
\hline & $(\%)$ & 55,6 & 33,3 & 11,1 & 100 \\
\hline \multirow{2}{*}{ Sobrepeso } & $\mathrm{N}$ & 16 & 7 & 4 & 27 \\
\hline & $(\%)$ & 59,3 & 25,9 & 14,8 & 100 \\
\hline \multirow{2}{*}{$\begin{array}{l}\text { Obesidade } \\
\text { Moderada }\end{array}$} & $\mathrm{N}$ & 14 & 10 & 6 & 30 \\
\hline & $(\%)$ & 46,7 & 33,3 & 20,0 & 100 \\
\hline \multirow{2}{*}{ Total Geral } & $\mathrm{N}$ & 40 & 23 & 12 & 75 \\
\hline & $(\%)$ & 53,3 & 30,7 & 16,0 & 100 \\
\hline
\end{tabular}

IMC-Índice de massa corporal.

Fonte: dados primários de pacientes com diabetes mellitus de unidades de saúde.

Nota-se que, em média, as pessoas com DM2 mais satisfatório em relação, aos que praticam que praticavam atividade física em um nível atividades físicas moderadas e aos que não mais elevado, apresentaram níveis de HbAlc praticam atividades física (Tabela 3).

Tabela 3. Distribuição do nível de atividade física de pessoas com Diabetes Mellitus tipo 2, conforme classificação do Questionário Internacional de Atividade Física, segundo dosagem laboratorial de HbA1c. São Paulo, 2014

\begin{tabular}{|c|c|c|c|}
\hline \multirow{2}{*}{ Atividade } & \multicolumn{3}{c|}{ HbA1c } \\
\cline { 2 - 4 } & Média & Desvio Padrão & CV(\%) \\
\hline Sedentário & 8,86 & 2,58 & $29,12 \%$ \\
\hline Ativo & 8,37 & 2,25 & $26,88 \%$ \\
\hline Muito Ativo & 7,98 & 1,81 & $22,68 \%$ \\
\hline Geral & 8,54 & 2,33 & $27,28 \%$ \\
\hline
\end{tabular}

HbAlc - hemoglobina glicada; CV - Coeficiente de variação; DP-Desvio padrão.

Fonte: dados primários de pacientes com diabetes mellitus de unidades de saúde 
Análise estatística apontou pelo teste qui- atendidos na UBS são mais sedentários quando quadrado, a diferença entre o nível de atividade comparados com os pacientes atendidos no física e a localidade de atendimento dos pacientes CEDM (Tabela 4). com diabetes $(\mathrm{p}=0,041)$. Ou seja,os pacientes

Tabela 4. Distribuição das pessoas com Diabetes Mellitus tipo 2, por local de atendimento, segundo nível de atividade física conforme Questionário Internacional de Atividade Física. São Paulo, 2014

\begin{tabular}{|ccc|c|c|c|}
\hline \multirow{2}{*}{ Localidade } & \multicolumn{5}{c|}{ Atividade } \\
Total \\
\cline { 3 - 6 } & & Sedentário & Ativo & Muito Ativo \\
\hline \multirow{2}{*}{ UBS } & $\mathrm{N}$ & 21 & 13 & 2 & 36 \\
\hline \multirow{2}{*}{ CEDM } & $(\%)$ & 58,3 & 36,1 & 5,6 & 100 \\
\hline \multirow{2}{*}{ Total Geral } & $\mathrm{N}$ & 19 & 11 & 10 & 40 \\
\hline & $(\%)$ & 47,5 & 27,5 & 25,0 & 100 \\
\hline & $\mathrm{N}$ & $4 \%$ & 24 & 12 & 76 \\
\hline
\end{tabular}

UBS - Unidade Básica de Saúde; CEDM - Centro de Especialidades em Diabetes Mellitus. Fonte: elaboração própria.

\section{DISCUSSÃO}

Nas ultimas décadas, o perfil demográfico da população mundial mudou drasticamente. Observado pela diminuição da taxa de fecundidade e maior esperança de vida, nota-se que o envelhecimento cresceu abruptamente.

Diante dessas mudanças, surge transformações nos indicadores epidemiológicos, evidenciando que em geral, as condições de saúde na população adulta e nos idosos são crônicas e múltiplas, perduram por vários anos e exigem acompanhamento das especialidades médicas e multiprofissionais permanente.
As condições de saúde crônicas são consideradas como verdadeiras epidemias, com estimativa de alcance de $19 \%$ da população mundial em 2050

e tem se tornado um sério desafio para a saúde pública. Dentre elas, o Diabetes Mellitus (DM) apresenta estimativas da ordem de $11.3 \%$ ou a $8^{\text {a }}$ posição mundial de prevalência em 2030. O DM tem sido caracterizado um problema de grande impacto quando comparado à distribuição das condições crônicas ${ }^{17}$.

Embora a legislação brasileira relativa aos cuidados com esta população seja avançada, a prática ainda não é satisfatória. $\mathrm{O}$ exemplo é a Estratégia de Saúde da Família (ESF), que 
presupõe por um modelo centrado no usuário e família. Um dos objetivos da ESF é facilitar à população atendimento com acompanhamento contínuo. Apesar da ampliação da ESF, que revelou a vulnerabilidade social da população idosa, a inserção da mesma nas redes de Assistência à Saúde ainda é incipiente ${ }^{19,20}$.

A ESF inserida no contexto da Atenção Básica (AB), garante resolutividade, de $85 \%$ dos problemas de saúde na comunidade de atenção, prestando cuidados com ações de promoção à saúde, prevenção de doenças, reduzindo ou evitando internações sensíveis ${ }^{21}$, à $A B$ e propiciando melhora da qualidade de vida da população ${ }^{22}$.

O Ministério da Saúde (MS), define AB como um conjunto de ações, de caráter individual ou coletivo, situadas no primeiro nível de atenção nos sistemas de saúde. No suporte e continuidade à atuação da $\mathrm{AB}$, o indivíduo é direcionado ao nível secundário da atenção à saúde. Este grupo de especialistas e com tecnologia intermediária (média complexidade) para acompanhamento. Ambos os níveis fazem parte junto com o terceiro nível (alta complexidade) na Rede de Atenção à Saúde (RAS) que propõe assegurar ao usuário o conjunto de ações e serviços que necessita com efetividade e eficiência, tentando superar a fragmentação da atenção e do gerenciamento nas Regiões de Saúde e aperfeiçoar o funcionamento político-institucional do $\operatorname{SUS}^{20,23}$.

A transição epidemiológica do Brasil sugere um déficit de ações de prevenção e controle das Doenças Crônicas Não-Transmissíveis (DCNT) devido, a necessidade de profissionais da saúde competentes e dispostos a mudar paradigmas culturais para a atuação eficaz. Assim, faz-se necessário o desenvolvimento de novos perfis profissionais. Avivar competências como o trabalho em equipe, ampliada no que diz respeito à rede como um todo e a integração de novas tecnologias nas rotinas de trabalho e na relação com os pacientes em todos os níveis da RAS ${ }^{23}$.

O MS atribuiu ao enfermeiro estratégias, com o objetivo de prevenir as DCNT e diminuir as possíveis complicações decorrentes do DM, a ele cabe desenvolver atividades educativas com os pacientes diabéticos; realizar consulta de enfermagem a pessoas com maior risco para diabetes tipo II e contribuir para o rastreamento; abordar fatores de risco, estratificando risco cardiovascular; orientar mudanças no estilo de vida e tratamento não medicamentoso; verificar adesão e possíveis intercorrências ao tratamento; estabelecer, junto à equipe, estratégias que possam favorecer a adesão da pessoa com DCNT, com os grupos de pessoas com DM, e avaliar os membros inferiores para identificação de potenciais sinais de risco, alerta do/para o desenvolvimento pé diabético ${ }^{24}$, acrescido de orientações para o autocuidado ${ }^{10}$.

Implementar práticas educativas e promotoras de saúde e ações de prevenção à agravos e complicações, lideradas por enfermeiros especialistas, têm demostrado resultados clínicos melhores no controle de condições crônicas, como o diabetes mellitus, favorem menos internações hospitalares, maior qualidade de vida a população e propor estratégias/modalidades de 
cuidado na perspectiva da atenção primária à saúde ${ }^{18}$.

O presente estudo aponta que a maioria dos participantes são mulheres adultas e idosas. Num contexto social de dupla função, cuidam da família e trabalham fora para ajudar nas despesas diárias, estudos semelhantes também demonstram que as mulheres acessam mais as unidades de saúde do que os homens ${ }^{25}$. Epidemiologistas explicam que a maior procura de mulheresocorre em virtude de que as mulheres se preocupam mais com a saúde e procuram o serviço de saúde com maior frequência quando comparado com os homens. Este fato reafirma a necessidade da reflexão sobre a saúde do homem pela falta de aderência destes, às práticas de manutenção da saúde na área de abrangência da ESF, que os torna mais vulneráveis às complicações das DCNT, como o DM2 e consequentemente a perda de sua funcionalidade global ${ }^{26,27}$.

Foi evidenciado ainda, que a maioria dos participantes apresentaram IMC alterado, com sobrepeso ou obesidade, isto constatado em ambos os serviços de saúde. Contudo, notou maior frequência de pessoas IMC alterados, nos participantes analisados na UBS, estes estão mais obesos em relação aos atendidos no CEDM, estatisticamente esta diferença é de $\mathrm{p}=0,894$.

Estudos revelam que quanto mais inativos o indivíduo maior IMC. O nível de atividade física é inversamente proporcional a presença/ quantidade gordura corporal e ao peso dos indivíduos ${ }^{27-29}$.
Quando comparados os níveis de HbAlc dos participantes, observou-se que os pacientes assistidos no CEDM, apresentaram melhor controle do DM em relação aos pacientes assistidos na UBS, com análise estatística significativa, $p=0,017$. Pesquisa realizada com pessoas com diabetes mellitus nos três níveis de atenção à saúde de município do interior paulista identificou também melhor controle do DM a partir dos níveis de HbAlc, no serviço secundário que na unidade básica de $\operatorname{saúde}^{27}$. Esses resultados se devem ao aparato deste serviço que permite ao paciente um controle melhor da doença, diagnóstico precoce, consultas com maior frequência, exames laboratoriais realizados em curto espaço de tempo, presença de equipe multiprofissional, com profissionais especializados em diferentes áreas, tais como: enfermeiro, nutricionista, oftalmologista, endocrinologista, fisioterapeuta, psicólogo, profissional de educação física. Isto permitindo acompanhamento da condição de saúde mais próxima $^{18,30}$.

Estudos mostram que quanto maior o nível de atividade física mais benefícios à saúde. A inatividade física, na população em geral, é uma situação preocupante ${ }^{4,27}$. Com os avanços tecnológicos e a carência de tempo, as pessoas estão desenvolvendo um estilo de vida com alto nível de sedentarismo, o que pode repercutir na elevação do IMC, podendo em médio ou longo prazo, resultar em distúrbios metabólicos, complicações e outras doenças ${ }^{13,27}$.

Este estudo mostrou que os pacientes que praticam atividade física em níveis mais elevados 
apresentaram níveis satisfatórios de $\mathrm{HbA1c}$, em ambas as unidades de saúde. Isto se dá devido aos efeitos produzidos pelo exercício físico, entre eles: aumento da sensibilidade à insulina, redução do peso corporal, favorecimento da resposta imune-antinflamatória ${ }^{29,31}$. A literatura apresenta estudos semelhantes, com resultados semelhantes, quando comparado atividade física e controle de doenças crônicas, de fato em ambos serviços os participantes não demonstram praticar atividade física suficiente, como parte essencial do tratamento e controle do DM.

Diante destes dados é importante considerar que os resultados desta pesquisa permitiram identificar que os indicadores de qualidade para avaliação do Programa de Prevenção e Controle do Diabetes, composto pelo o IMC, resultados do HbAlc e nível de atividade física não estão em consonância com as recomendações e diretrizes internacionais e nacionais, em especial os apontados pela Sociedade Brasileira de Diabetes e referendados pelo do Ministério da Saúde.

Em relação ao IMC e o nível de atividade física pode-se sugerir que há um desequilíbrio no balanço energético, com uma ingesta mais elevada que a queima calórica.

Recomenda-se que o valor da HbA1c, em pessoas com DM, seja de 6,5\% e IMC $<25 \mathrm{~kg} /$ $\mathrm{m}^{2}$ e os resultados apontam para um valor acima em todos os pacientes avaliados ${ }^{25}$. Desta forma as ações e objetivos propostos pela OMS a serem desempenhados pelos gestores municipais ressaltam a relevância de um contexto social, econômico e ambiental favorável para os indivíduos aderirem a estilos de vida mais benéfico, obtidos com ações efetivas para reduzir consideralmenteas mortes e as doenças crônicas no cenário mundial. A adoção de mudanças de comportamentos inseridos nas ações de promoção à saúde, medidas preventivas constitui um grande desafio na atualidade.

Os pacientes atendidos no Centro de Referência de Diabetes, que possui equipe multiprofissional habilitada, apresentaram melhor desempenho no nível de atividade física e melhores níveis glicêmicos obtidos pelo exame da hemoglobina glicada quando comparados aos pacientes acompanhados na Unidade Básica de Saúde. Este aspecto reforça a importância da implementação e aprimoramento de uma equipe multiprofissional para o desempenho no estímulo para a mudança de hábitos de vida e a necessidade de rever as ações de prevenção das complicações secundárias das doenças crônicas. Evidências internacionais demonstram os resultados e impactos positivosdos serviços de saúde efetivamente integrados sob o modelo de redes, podendo salientar alguns deles: melhor saúde; maior qualidade na atenção às necessidades dos usuários; menor desperdício; maior eficiência e experiência mais satisfatória, aos pacientes e aos profissionais ${ }^{20,27}$.

Mensurar comportamentos pode tornar a pesquisa uma atividade insana, recomendar padrões de conduta requer antes de tudo compreender a dimensão do ser humano e da saúde na multifatoriedade. Tornar-se um profissional que atua na mudança de hábitos exige competência cientifica, técnica e engajamento político e ético ${ }^{31}$.

A OMS vem propondo ações e objetivos gerais a serem desempenhados pelos gestores municipais. 
Nesse sentido, é ressaltada a importância de um contexto social, econômico e ambiental favorável para que os indivíduos assumam práticas saudáveis.

As limitações do estudo consistiram em um universo reduzido de observação da população, especialmente na unidade de atenção básica, recursos documentais para análise das variáveis e associações propostas. Evidenciou-se também, dificuldades de implementação de políticas publícas saudáveis no território adstrito.

\section{CONCLUSÕES}

A partir do estudo foi possível concluir que tanto a população atendida na atenção básica quanto as atendidas no nível secundário, não demonstram realizar a prática de atividade física suficiente, como parte integrante do tratamento e controle do DM.

As pessoas atendidas no centro especializado apresentaram melhor desempenho no nível de atividade física e melhores níveis glicêmicos obtidos pelo exame da HbA1 c quando comparados às acompanhadas na UBS. Este aspecto reforça a importância da capacitação dos profissionais para o desempenho do estímulo à mudança de hábitos de vida e a necessidade de considerar as ações de prevenção das complicações secundárias das doenças crônicas.

O cuidado integral, as ações interdisciplinares, a organização na Rede de Atenção à Saúde na linha de cuidado em diabetes, são fatores que associados permitem a melhora na adesão terapêutica, econtrole mais adequado dos níveis glicêmicos.

Conflito de interesses: Os autores declaram que não houve conflito de interesses.

\section{REFERÊNCIAS}

1. American Diabetes Association. Standards of medical care in diabetes. Diabetes Care. 2017; 40 Suppl 1: S5-S17.

2. Church T. Exercise in obesity, metabolic syndrome, and diabetes. Prog Cardiovasc Dis. 2011; 53(6): 412-8. http://dx.doi.org/10.1016/j.pcad.2011.03.013

3. Umpierre D, Ribeiro PA, Kramer CK, Leitão CB, Zucatti AT, Azevedo MJ, et al. Physical activity advice only or structured exercise training and association with HbA1c levels in type 2 diabetes: a systematic review and meta-analysis. JAMA. 2011; 305(17): 1790-9. http://dx.doi.org/10.1001/jama.2011.576

4. Sánchez RT, Molina EM, Gómez-Ortega OR. Intervenciones de enfermería para disminuir la sobrecarga en cuidadores: un estudio piloto. Rev Cuid. 2016; 7(1): 1171-84. http://dx.doi.org/10.15649/cuidarte.v7i1.251

5. Diabetes Atlas Seventh Edition. International Diabetes Federation (IDF). Bruselas, Bélgica; 2015.

6. Faria HTG, Rodrigues FF, Zanetti ML, Araújo MFM, Damasceno MMC. Fatores associados à adesão ao tratamento de pacientes com diabetes mellitus. Acta Paul Enferm. 2013; 26(3): 231. http://dx.doi.org/10.1590/S0103-21002013000300005

7. Cunha M, Chibante R, André S. Suporte social, empowerment e doença crónica. R. Port. Enferm. Saúde Mental. 2014; 1 (n. ${ }^{\circ}$ esp.): $21-6$.

8. Leite ES, Lubenow JAM, Moreira MRC, Martins MM, Costa IP, Silva AO. Cienc Cuid Saude. 2015; 14(1): 822 9. http://dx.doi.org/10.4025/cienccuidsaude.v14i1.21353

9. Arteaga A, Cogollo R, Muñoz D. Apoyo social y control metabólico en la diabetes mellitus tipo 2. Rev Cuid. 2017; 8(2): 1668-76.

http://dx.doi.org/10.15649/cuidarte.v8i2.405

10. Pasqualotto KR, Alberton D, Frigeri HR. Diabetes mellitus e Complicações. J. Biotec. Biodivers. 2012; 3(4).

11. Batista F, Magalhães AA, Gamba M, Nery C, Cardoso C. Ten years of a multidisciplinary diabetic foot team approach in Sao Paulo, Brazil. Diabet Foot \& Ankle. 2010; 1: 3. http://dx.doi.org/10.3402/dfa.v1i0.5203

12. Thomaz PMD, Costa THM, Silva E, Hallal PC. Fatores associados à atividade física em adultos. Rev. Saúde Pública. 2010; 44(5): 894-900. http://dx.doi.org/10.1590/S0034-89102010005000027

13. Ramalho JR, Lopes ACS, Toledo MTT, Peixoto SV. Physical activity levels and factors associated with the sedentary lifestyle of users of a basic health unit in Belo Horizonte, Minas Gerais. Rev Min Enferm., 2014; 18(2). http://dx.doi.org/10.5935/1415-2762.20140032 
14. Pardini R. Validação do questionário internacional de nível deatividade física (IPAQ - versão 6): estudo piloto em adultos jovens brasileiros. Rev. Bras. Ciên. e Mov. 2001; 9(3): 45-51.

15. The PLOS Medicine Editors. Observational Studies: Getting Clear about Transparency. PLOS Medicine. 2014. https://doi.org/10.1371/journal.pmed.1001711

16. Jurakić D, Pedišić Ž, Andrijašević M. Physical Activity of Croatian Population: Cross-sectional Study Using International Physical Activity Questionnaire. Croat Med J. 2009; 50 (2):165-73. http://dx.doi.org/10.3325/cmj.2009.50.165

17. Magalhães MN, Lima ACP. Noções de Probabilidade e Estatística. São Paulo: EDUSP; 2005.

18. Goulart FAA. Doenças Crônicas Não Transmissíveis: Estratégias de controle e desafios para os sistemas de saúde. Brasília. 2011. 34 p.

19. Ministério da Saúde, Brasil. Secretaria de Atenção à Saúde, Departamento de Atenção Básica. Cadernos de Atenção Básica. Estratégias para o cuidado da pessoa com doença crônica. n. 35. Brasília; 2013.

20. Ministério da Saúde, Brasil. Portaria n ${ }^{\circ} 4279$, de 30 de dezembro de 2010. Estabelece diretrizes para a organização da Rede de Atenção à Saúde no âmbito do Sistema Único de Saúde (SUS). Diário Oficial da União. 2010 dez.

21. Sousa N, Rehem T, Santos W Silva, Santos C. Internações sensíveis à atenção primária à saúde em hospital regional do Distrito Federal. Rev. Bras. Enferm., 2016; 69(1): 11825. http://dx.doi.org/10.1590/0034-7167.2016690116i

22. Borba AK, Marques AP, Ramos VP, Leal MC, Arruda Ilma KG, Ramos RS. Fatores associados à adesão terapêutica em idosos diabéticos assistidos na atenção primária de saúde. Ciênc. Saúde Coletiva. 2018; 23(3): 95361. https://doi.org/10.1590/1413-81232018233.03722016

23. Ministério da Saúde, Brasil. Secretaria de Atenção à Saúde; Departamento de Atenção Básica. Cadernos de Atenção Básica, Diabetes Mellitus. n. 36. Brasília; 2013.

24. Oliveira PS, Bezerra EP, Andrade LL, Gomes PLF, Soares MJGO, Costa MML. Practice nurse family health strategy in the prevention of diabetic foot. J. res.: fundam. care. online. $2016 ; 8(3)$ : 4841-9.

http://dx.doi.org/10.9789/2175-5361.2016.v8i3.48414849

25. Sociedade Brasileira de Diabetes, Brasil. Diretrizes da Sociedade Brasileira de Diabetes (2015-2016). São Paulo; 2016.

26. Figueiredo WS, Schraiber LB. Male users' and primary care services health professionals' conceptions of gender and possible impacts on men's health, São Paulo, Brazil. Ciênc. Saúde Coletiva. 2011; 16 Suppl 1: 935-44. http://dx.doi.org/10.1590/S1413-81232011000700025

27. Souza J, Baptista MH, Gomides D, Pace AE. Adherence to diabetes mellitus care at three levels of health care. Esc. Anna Nery. 2017; 21(4):1-9.

http://dx.doi.org/10.1590/2177-9465-ean-2017-0045
28. Ferreira SRG. Atividade física no Diabetes tipo 1 e 2: Bases fisiopatológicas, importância e orientação. Tratamento do Diabetes: Abordagens Educacionais e de Alterações no Estilo de Vida. Em: e-book 2.0. Sociedade Brasileira de Diabetes Diabetes na prática clínica. Módulo 3 Cap 8. Brasília, Brasil; 2015. Módulo 3 - Capítulo 8.

29. Gonçalves HR, Gonçalves LA, Costa TA, Goulart MI. Associação entre IMC e Atividade Física em Adultos de Diferentes Níveis Socioeconômicos. Rev. Saúde e Pesquisa. 2011; 4(2): 161-8.

30. Interial MG, Campillo C, Aguilar I. Análisis comparativo del programa de educación en diabetes mellitus de México y Cuba. Rev Cuid. 2013; 4(1): 516-22.

http://dx.doi.org/10.15649/cuidarte.v4i1.12

31. Codogno JS, Fernandes RA, Sarti MF, Freitas FI, Monteiro H. The burden of physical activity on type 2 diabetes public healthcare expenditures among adults: a retrospective study. BMC Public Health. 2011; 11(275): 1-7. https://doi.org/10.1186/1471-2458-11-275 